\title{
RFID AC Current Sensing Technique
}

\author{
Irfan Ullah, Member, IEEE, Robert Horne, Benito Sanz-Izquierdo, and \\ John C. Batchelor Senior Member, IEEE
}

\begin{abstract}
The mechanism is described by which a passive UHF RFID tag coupled with a tuning circuit is integrated with a current transformer for sensing ac current in an electrical wire for smart power monitoring of individual appliances. A capacitance change in the tuning circuit results from a reverse bias voltage from the current transformer. The tuning circuit reactance is detected by a capacitance sensing RFID tag and the value is transmitted as a 5-bit sensor code which is directly related to the ac current drawn by an electrical load. The passive tag harvests energy and offers an innovative solution for energy management in future smart homes and for industry 4.0. As well as indicating current level, the technique can also be used to detect the ON and OFF state of an electrical device and is demonstrated to work for a rapidly switching load. The sensor is tuned for EPC Class 1 Generation 2 UHF RFID readers at $868 \mathrm{MHz}$.
\end{abstract}

\section{Index Terms}

RFID sensing, wireless sensors, Internet of Things, self-tuning tags, smart home application, industry 4.0 .

\section{INTRODUCTION}

Global energy demand is set to grow by more than a quarter by 2040 with electrical contribution increasing twice as fast as the overall demand [1]. The increase in electrical demand will put pressure on generators with associated greenhouse gas emissions [2]. Energy-saving solutions including smart electricity metering can be exploited to reduce overall electricity demand and these can be employed in houses or apartments for tracking real-time power consumption [3]. Such smart technologies would help consumers simultaneously to monitor the energy consumed

The INCASE project is part-funded by the European Commission as part of the 2Seas Intereg V Programme.

I. Ullah, R. Horne, B. Sanz-Izquierdo and J. C. Batchelor are with the School of Engineering and Digital Arts, University of Kent, Canterbury CT2 7NT, UK. (e-mail: \{iu22, r.j.horne, b.sanz, j.c.batchelor\}@kent.ac.uk). 
by multiple individual appliances, reduce usage, and to wirelessly report readings to a data acquisition module. However, installing smart metering is expensive and gives only overall intake. Developing new passive, affordable and easy-to-install technologies could play a valuable role in multiple appliance monitoring. UHF RFID systems potentially offer a sensing solution based on low cost tags with a single reader [4]-[6].

There have been RFID based solutions proposed for the sensing of home electrical devices [7]-[9] but which require the integration of an impulse acquisition circuit with the RFID tag. This needs an external power supply and leads to high maintenance costs. Furthermore, they only have the ability to detect the transition between On and Off states and are ill-suited for real-time load current sensing. Alternatively, [10], [11] report ZigBee-based wireless current transformers with complex architectures including a sensing platform which do not require a mains voltage ac-dc converter but do include the complexity of a signal processing circuit, an analogue-todigital converter and a wireless transmission module, which increases the expense. Alternatively, the Monjolo energy-harvesting energy meter does not require a mains ac-dc converter but still involves the complexity of an energy harvesting IC, a microcontroller, an IEEE 802.15.4 compatible radio and FRAM (ferroelectric random access memory) [12]. Finally, a new energy harvesting technique for capturing power from ac power lines using a miniature linear permanent magnet synchronous generator is described in [13]. However, such a device is most useful for monitoring high power consumption in emerging smart grids.

This paper presents a new method for sensing ac current from an electrical cable based on a passive capacitive sensing UHF RFID tag combined with a tuning circuit and a Current Transformer (CT). The tag structure is less complex than the alternatives, and provides a cost effective solution for power monitoring when many tags on individual appliances communicate wirelessly with a single RFID reader. The tag generates a sensor code to represent the load current and offers a solution for small current measurements in the range of 1.5-10 A without requiring a microcontroller, and which harvests energy from the interrogator radio wave. Section II describes the sensing principle, Section III presents experimental measurements and results, and Section IV concludes the paper.

\section{SYSTEM DESCRIPTION}

The current sensing tag incorporates a self-tuning RFMicron Magnus S2 transponder chip which provides two key features: a 5-bit sensor code and a 5-bit on-chip RSSI (Received 


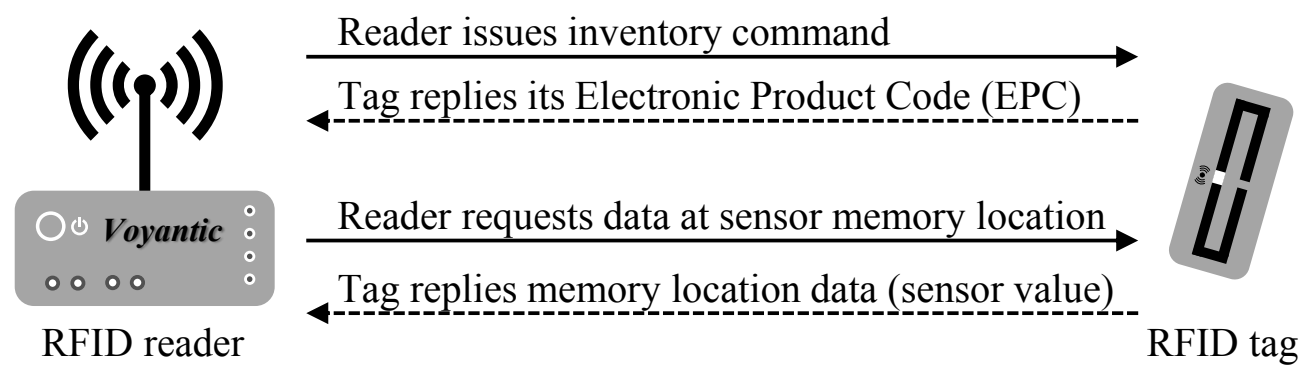

Fig. 1. RFID reader communication and retrieval of tag sensor codes [16].

Signal Strength Index) code which can be transmitted to an external RFID reader [14], [15]. The sensor code indicates the impedance mismatch between the chip and the tag antenna, while the RSSI code relays the strength of the incoming signal from the reader. Fig. 1 illustrates how a reader communicates with the RFID tag and retrieves its sensor codes using standard GEN2 READ commands [16]. While a tag is receiving transmitted power from the dedicated reader, it writes sensor values into its reserved memory bank.

The schematic diagram of the tag system is shown in Fig. 2 and comprises of three elements: (i) a Current Transformer (CT), (ii) a tuning circuit, and (iii) a capacitance sensing passive UHF RFID tag. The CT is used to detect the ac current from a cable and transforms it into dc voltage which controls the reactance of a tuning circuit. This effects a reactance change at the capacitance sensing RFID chip which generates a proportionate sensor code. The changes in the tuning circuit capacitance as a function of the reverse bias voltage from the CT are proportional to the sensor code as the transformer has a linear relationship between primary current and secondary voltage, and as the mains frequency is fixed, the secondary voltage relates to the primary ac magnitude in the electrical load.

\section{A. Current Transformer (CT)}

An off-the-shelf split-core CT with a full bridge rectifier [27] was used to sense input currents of 0-10 A ac and transform them to $0-10 \mathrm{~V}$ dc voltage output with a stated full-scale accuracy of $\pm 2 \%$ at frequencies of $50 / 60 \mathrm{~Hz}$. The CT also includes a burden resistor, a low-pass filter and a zener diode. The burden resistor across the bridge rectifier provides the output voltage for measurement purposes while the filter capacitor parallel to the output attenuates higher frequency components. The bi-directional zener diode maintains the unloaded voltage output up to a $15 \mathrm{~V}$ 


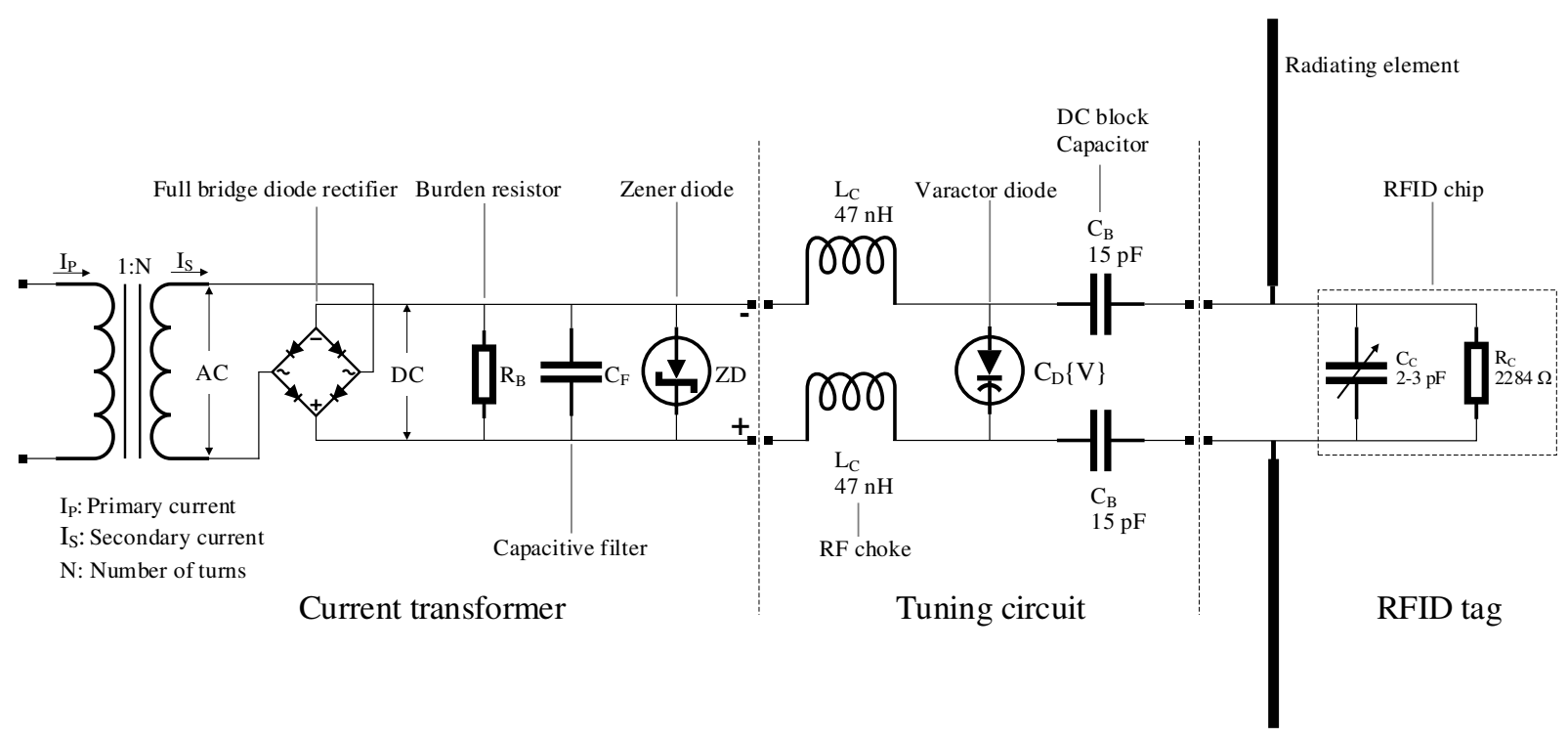

Fig. 2. Schematic diagram overview of the suggested RFID ac current sensing tag from an ac electrical cable.

dc limit and prevents the development of high voltage spikes across the output. Two M3 screw terminals beneath a removable cover can be connected to the output leads.

\section{B. Tuning Circuit}

The tuning operating circuit in Fig. 2 is composed of two inductors, a tuning varactor diode and two capacitors. The reverse-biased varactor diode [18] capacitance $V_{D}\{V\}$ is a function of the applied CT voltage $V$. The diode typically requires a reverse bias of $0-20 \mathrm{~V}$ to produce capacitance in the range of $0-3.3 \mathrm{pF}$, though the diode capacitance starts to saturate at $10 \mathrm{~V}$. The inductors isolate the RF from the tag antenna from the CT secondary. The $15 \mathrm{pF}$ capacitors are used to block the CT output dc voltage from the input of the tag chip, as shown in Fig. 3 and the relationship between the capacitance and the reverse bias voltage taken from [19] is illustrated in Fig. 4. Therefore, the equivalent capacitance $\left(C_{e q}\right)$ of the tuning circuit is equal to the total series capacitance. The upper or lower output capacitance of the tuning circuit can be determined through different values of the lumped elements, i.e. an increased dc block capacitor value $C_{B}$ will lead to an increase in the overall tuning circuit capacitance:

$$
\frac{1}{C_{e q}}=\frac{1}{C_{B}}+\frac{1}{C_{D}\{V\}}+\frac{1}{C_{B}}
$$


The tag antenna and tuning circuit layout are shown in Fig. 5. Two $1 \mathrm{~mm}$ wide, $20 \mathrm{~mm}$ long copper straps with a $4 \mathrm{~mm}$ gap are used to connect the CT to the lumped elements of the tuning circuit. The tuning circuit uses 0603 surface mount components with a pitch of $0.5 \mathrm{~mm}$ and was fabricated along with the RFID tag on a $0.14 \mathrm{~mm}$ thick flexible Mylar substrate with a copper layer of $0.04 \mathrm{~mm}$ thickness.

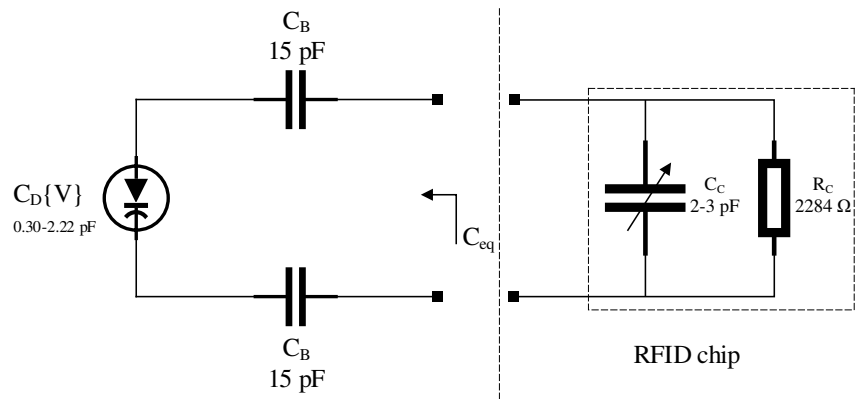

Fig. 3. The configuration of dc block capacitors and varactor diode in the tag tuning circuit.

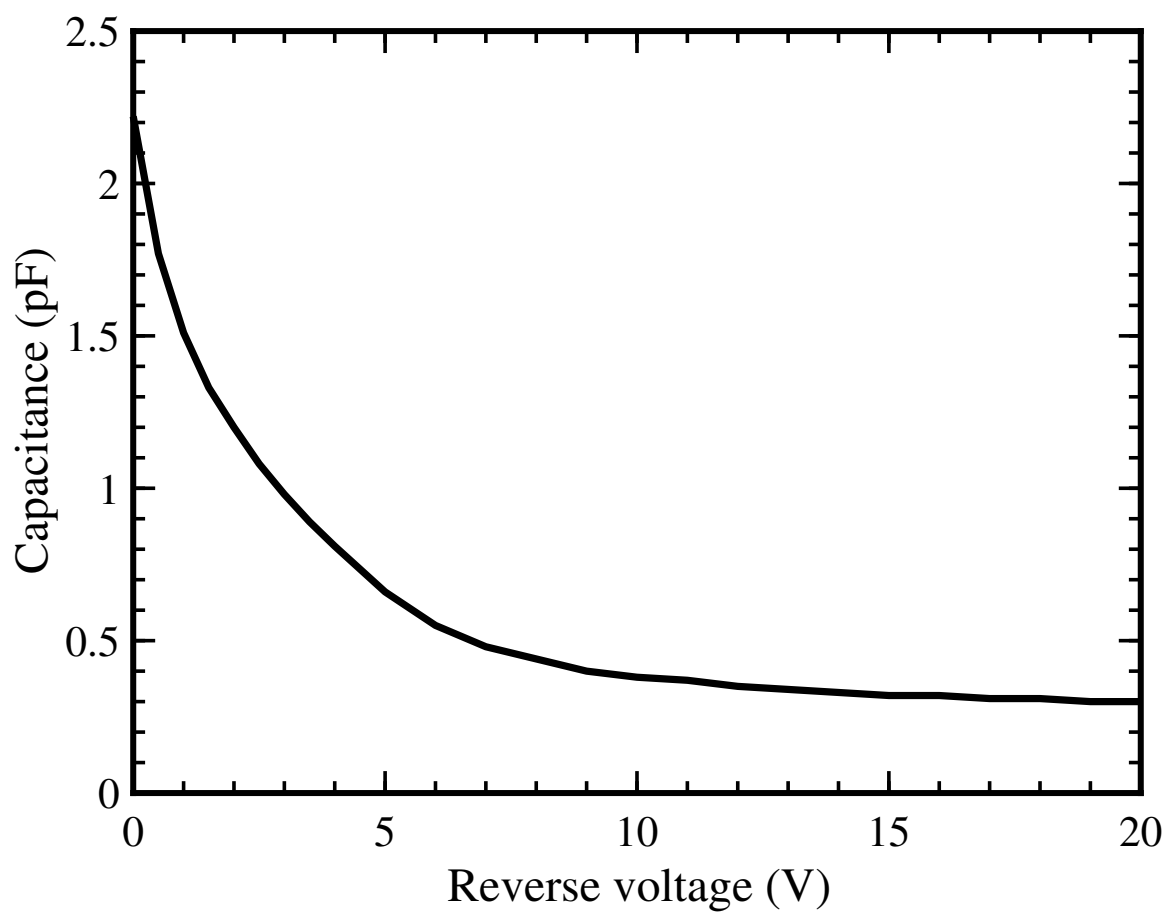

Fig. 4. Voltage to capacitance ratio of the implemented tuning element in the tuning circuit [19]. 


\section{RFID Tag Design}

The RFID dipole antenna in Fig. 5(a) operates at $868 \mathrm{MHz}$ and is similar to the structure presented in [20]. The tag was simulated in the electromagnetic software Computer Simulation Technology (CST) Microwave Studio [21] and the length of the antenna feed loop was adjusted to shift its resonance frequency. The RFMicron Magnus S2 chip required turn-on power is -16.1 $\mathrm{dBm}$ and the tuning capacitance range is $2-3 \mathrm{pF}$ in parallel with $2284 \Omega$. The chip covers all Worldwide RFID frequency bands (860-960 MHz) and can vary its capacitance between 2-3 $\mathrm{pF}$ in the presence of a detuning element. The total load capacitance for the antenna model is [14]:

$$
C_{T}(n)=C_{\min }+n C_{o}
$$

where $C_{T}(n)$ is the total capacitance, $C_{\min }$ is the minimum capacitance of $2 \mathrm{pF}$ of the chip, $\mathrm{n}$ is the sensor value in the range of $0-31$ and $C_{o}$ is a tunable step $(1 \mathrm{pF} / 31)$ within the $1 \mathrm{pF}$ tunable range. Therefore, for simulation, the chip impedance was fixed to $1.63 \Omega-\mathrm{j} 61.07 \Omega$, at $868 \mathrm{MHz}$ (corresponding to $3 \mathrm{pF}$ ). The tag was designed on a Mylar substrate with dielectric constant $\left(\varepsilon_{r}\right)$ of 2.8 , loss tangent $(\delta)$ of 0.003 , and with a total height of $0.18 \mathrm{~mm}$ including the copper thickness of $0.04 \mathrm{~mm}$. The tag performance was simulated for two scenarios: (i) in the absence of detuning elements and (ii) including the detuning elements, i.e, a total capacitance of 2.1-3 pF. Fig. 6 illustrates the simulated effect of varying applied terminal capacitance on the reflection coefficient $S_{11}$ of the tag antenna in Fig. 5. In the absence of the auto-tuning function, increasing the applied capacitance to $1 \mathrm{pF}$ causes the antenna resonance to drop to $800 \mathrm{MHz}$. Furthermore, the simulated results in Fig. 7 show the variation of antenna admittance with a change of applied capacitance. The relation between the chip and the antenna susceptance is:

$$
\left|B_{C}(n)+B_{A}(\Psi)\right| \rightarrow 0
$$

where $B_{C}$ is the susceptance of the chip, $B_{A}$ is the susceptance of the tag antenna, and $\Psi$ is an external reactance $(0.1-1 \mathrm{pF})$. When tag antenna susceptance changes as a result of the applied reactance this will be compensated by the self-tuning chip and returned in the form of the sensor code $(n)$. 


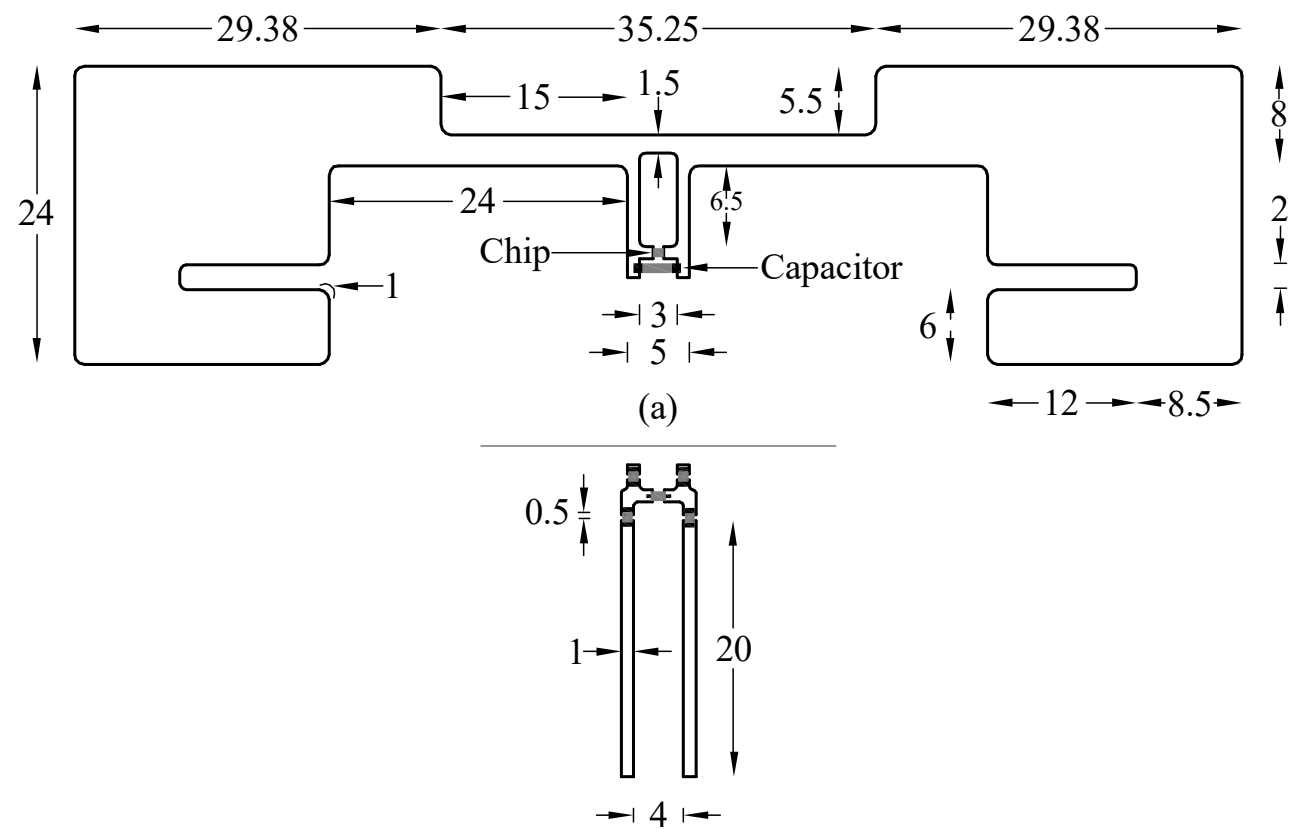

(b)

Fig. 5. (a) RFID tag design, (b) tuning circuit design. (All dimensions in mm)

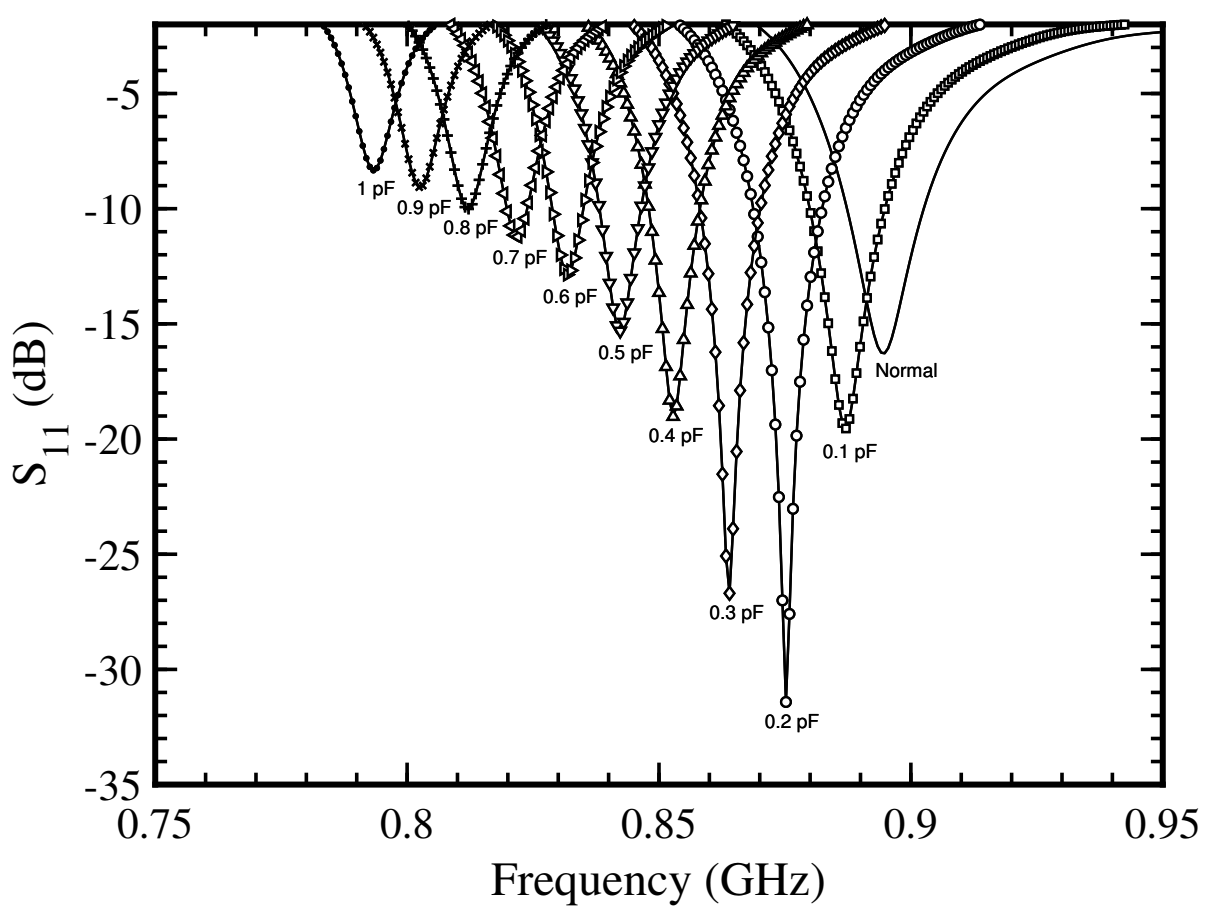

Fig. 6. CST simulated power reflection coefficient of the RFID tag antenna due to varying capacitor value across its terminals. 


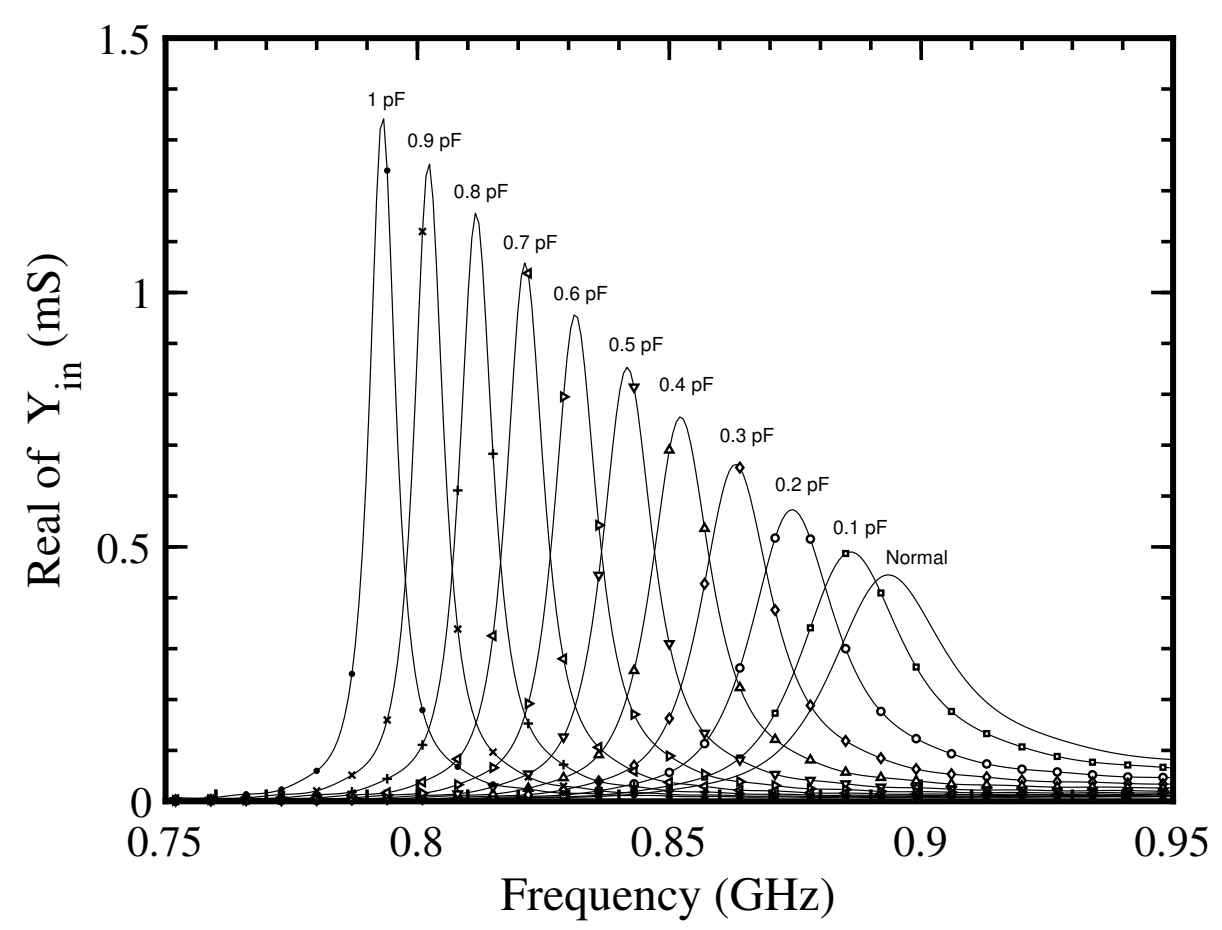

(a)

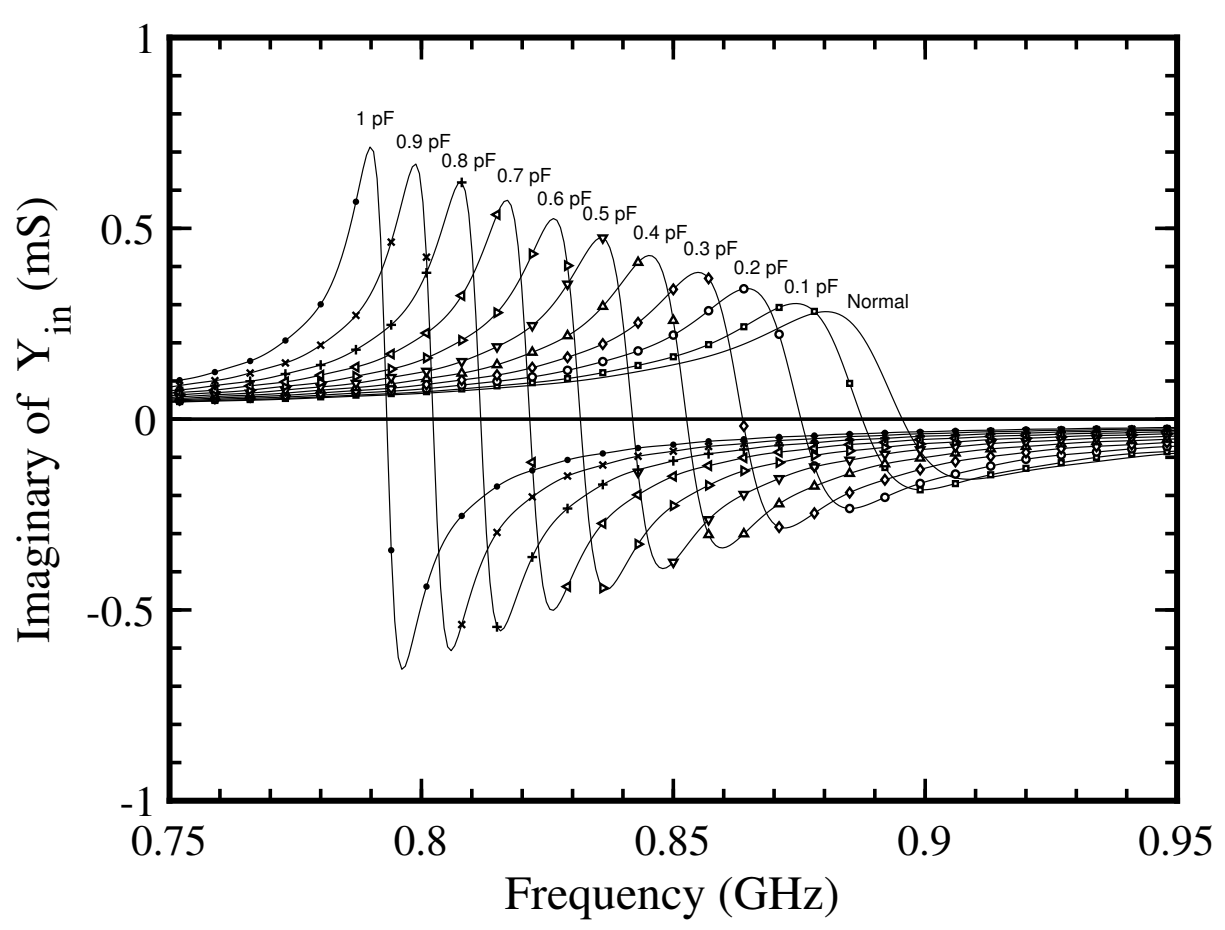

(b)

Fig. 7. CST simulated power input admittance of the capacitance sensing RFID tag (a) Real input admittance, (b) Imaginary input admittance. 


\section{Measurements AND Results}

\section{A. Capacitance To Sensor Code Relationship}

To assess the performance, ten tag samples were fabricated on the Mylar substrate. Ten different capacitors in the range of $0.1-1 \mathrm{pF}$ were placed across at the terminals of each tag antenna, as shown in Fig. 5(a). The tags were interrogated by a UHF reader Tagformance Pro System, comprising of a linear polarized antenna of $6 \mathrm{dBi}$ gain and placed a fixed distance of 30 $\mathrm{cm}$ away from the sensor [22]. The tag is capable of operation at distances greater than $30 \mathrm{~cm}$, and the calibrated Tagformance system was able to assess the maximum read distance achievable in each measurement. The set up was also able to record the sensor code as well as provide tag threshold power analysis.

Firstly, the tags were measured by the Voyantic reader to obtain the sensor code values, Fig. 9. These results demonstrate a linear relationship between the sensor value and the applied capacitance. Sensor code values of 26 and 3 were returned for zero applied reactance, and $1 \mathrm{pF}$ respectively. Increasing capacitance over $1 \mathrm{pF}$ falls outside the tag tuning capability and the tag frequency would deviate from $868 \mathrm{MHz}$. An RFID tag was then connected to the tuning circuit, Fig. 8. A voltage of $0-10 \mathrm{~V}$ dc was applied to the copper straps of the tuning circuit and the sensor code was measured. The total equivalent capacitance of the varactor tuning circuit

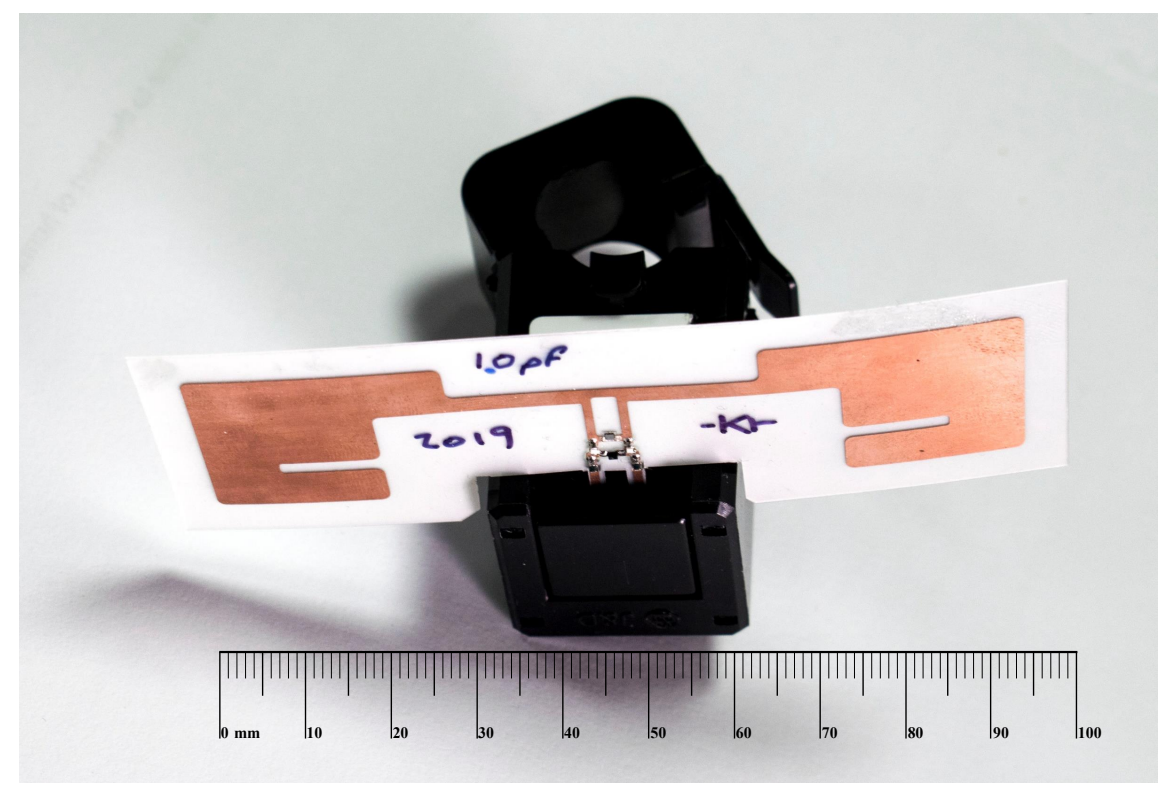

Fig. 8. A final tested prototype of the RFID ac current sensor. 


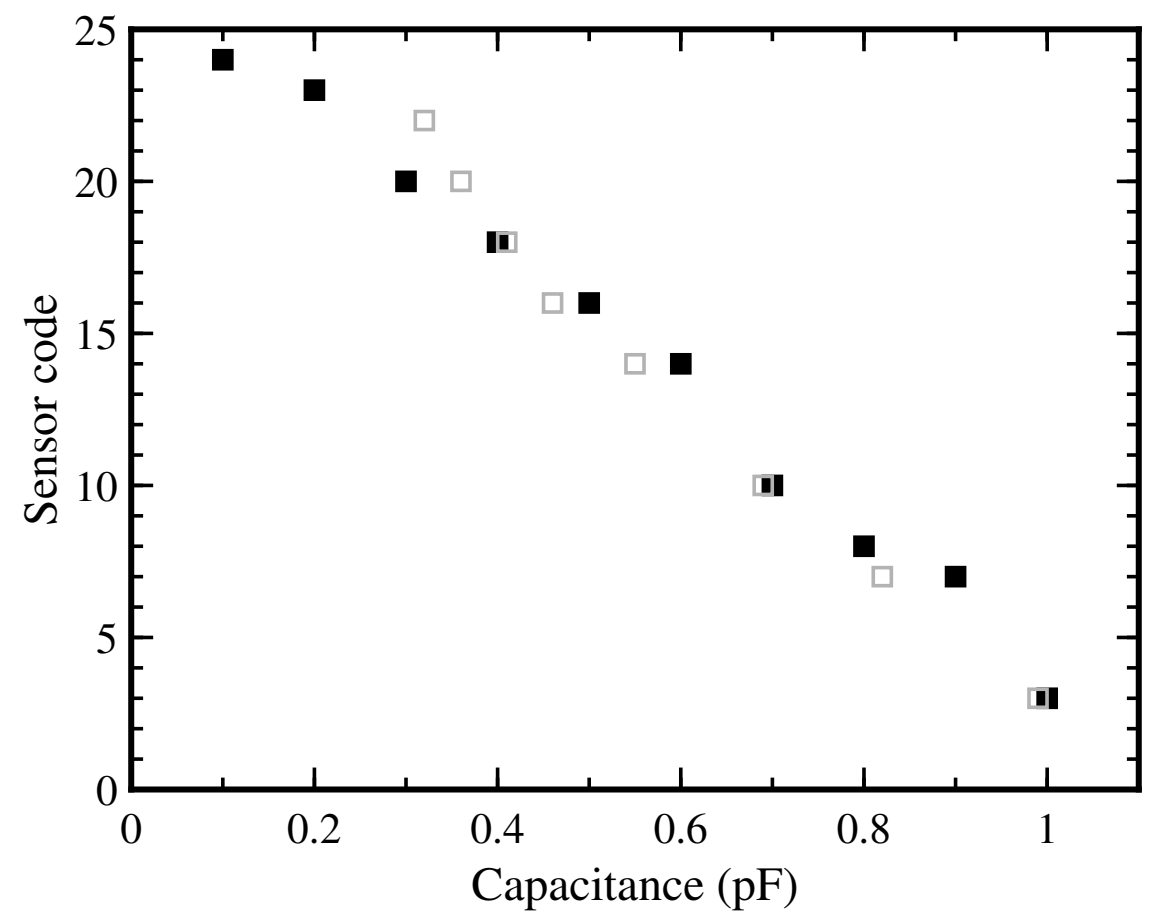

Fig. 9. Measured sensor code of a self-tuning tag at $868 \mathrm{MHz}$. Black square markers: tag loaded with lumped capacitance in the range of $0.1-1 \mathrm{pF}$. Grey line square markers: tag with tuning circuit.

was calculated with (1) and is compared with the measured sensor codes in Fig. 9. The tag response when connected to lumped capacitances is seen to agree strongly with the response to the tuning circuit reactance indicating the transducer functioned as expected. A slight difference in response of the tuning circuit to the lumped values near $0.3 \mathrm{pF}$ arises from the non-linear junction capacitance of the varactor (Fig. 4). Although this non-linearity can be compensated for, it does influence the lower limit of the sensing range. However, the overall agreement between the two measurements can be seen to be very good without the need for any complex calibration.

\section{B. Power Cable Measurement}

In the final set of experiments, the RFID tag along with the tuning circuit was linked with the CT using the copper straps and the M3 screw terminals. The magnetic circuit of the CT encircled a single split-core cable that was enclosed in an insulating box as the CT must be installed on a single live or neutral current carrying wire, Fig. 10. The sensor response was measured with the Tagformance Pro System as described earlier. A switchable electrical heater with an adjustable 


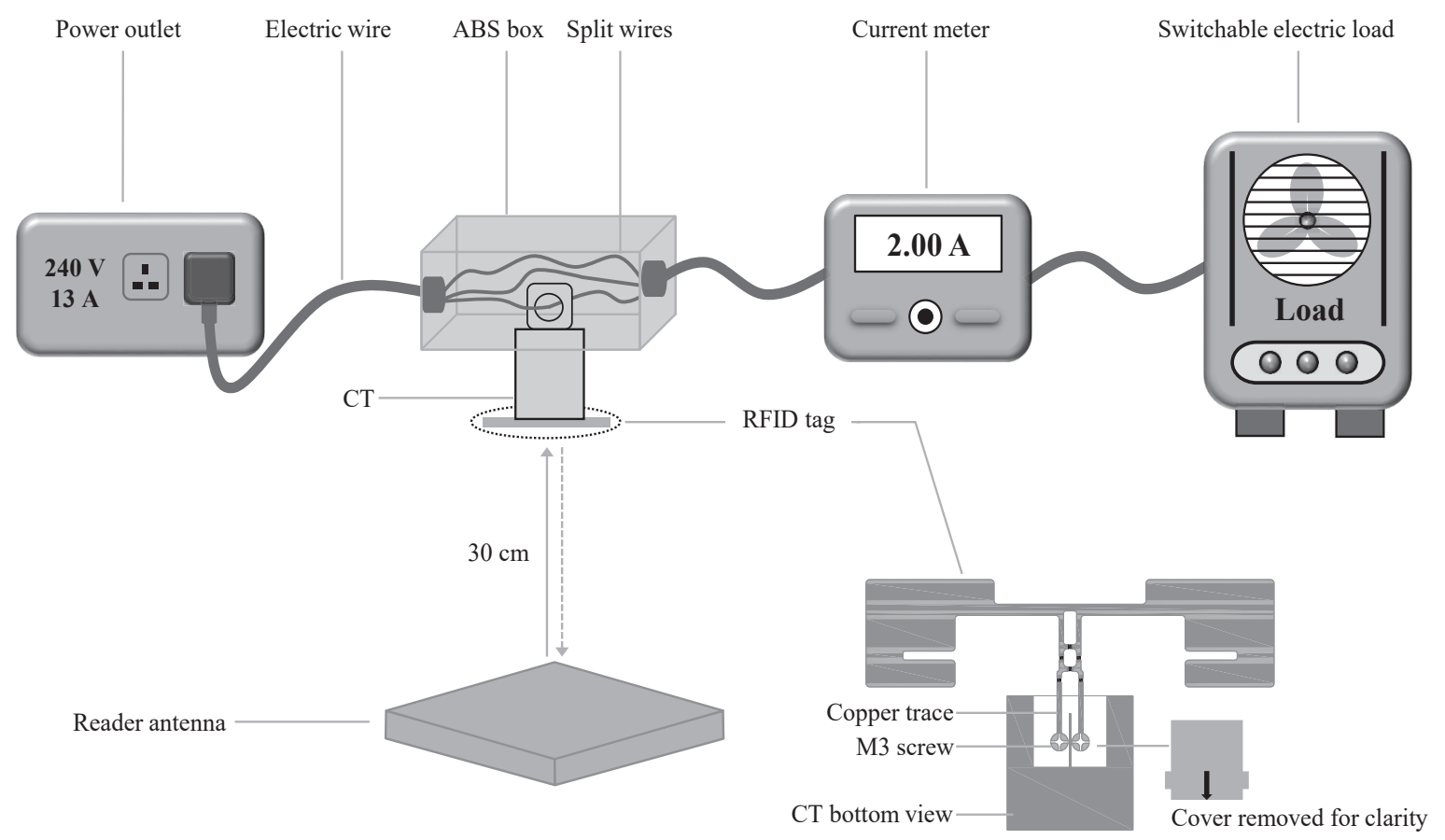

Fig. 10. Block diagram of the measurement setup.

load was used to test the system. The sensor tag was placed $30 \mathrm{~cm}$ away from the dedicated reader antenna to obtain calibrated read range results and sensor codes. A commercial Brennenstuhl PM 231 E current meter with $\pm 1 \%$ accuracy [23] was used to benchmark the tag results.

\section{Current To Sensor Code Relationship}

Measurements were carried out for load currents ranging from 0-10 A with a step of $0.1 \mathrm{~A}$ and each measurement was taken ten times for a signal tag. Fig. 11 illustrates the mean values of the measured sensor code and also indicates the spread for each point. The experiment was repeated with a second tag and the response was consistent. The results show that the proposed sensor can detect in the range of 1.5-10 A with a resolution of $0.5 \mathrm{~A}$. As this passive sensor operates on the principle of self-tuning variable capacitance, there is a limited tuning window to the capacitance tuning before the value starts to saturate. Therefore, where less than $1.5 \mathrm{~A}$ or more than $10 \mathrm{~A}$ ac current measurement is required, then the tuning circuit could be adjusted or a current transformer with a different turns ratio could be employed. To assess the effect 


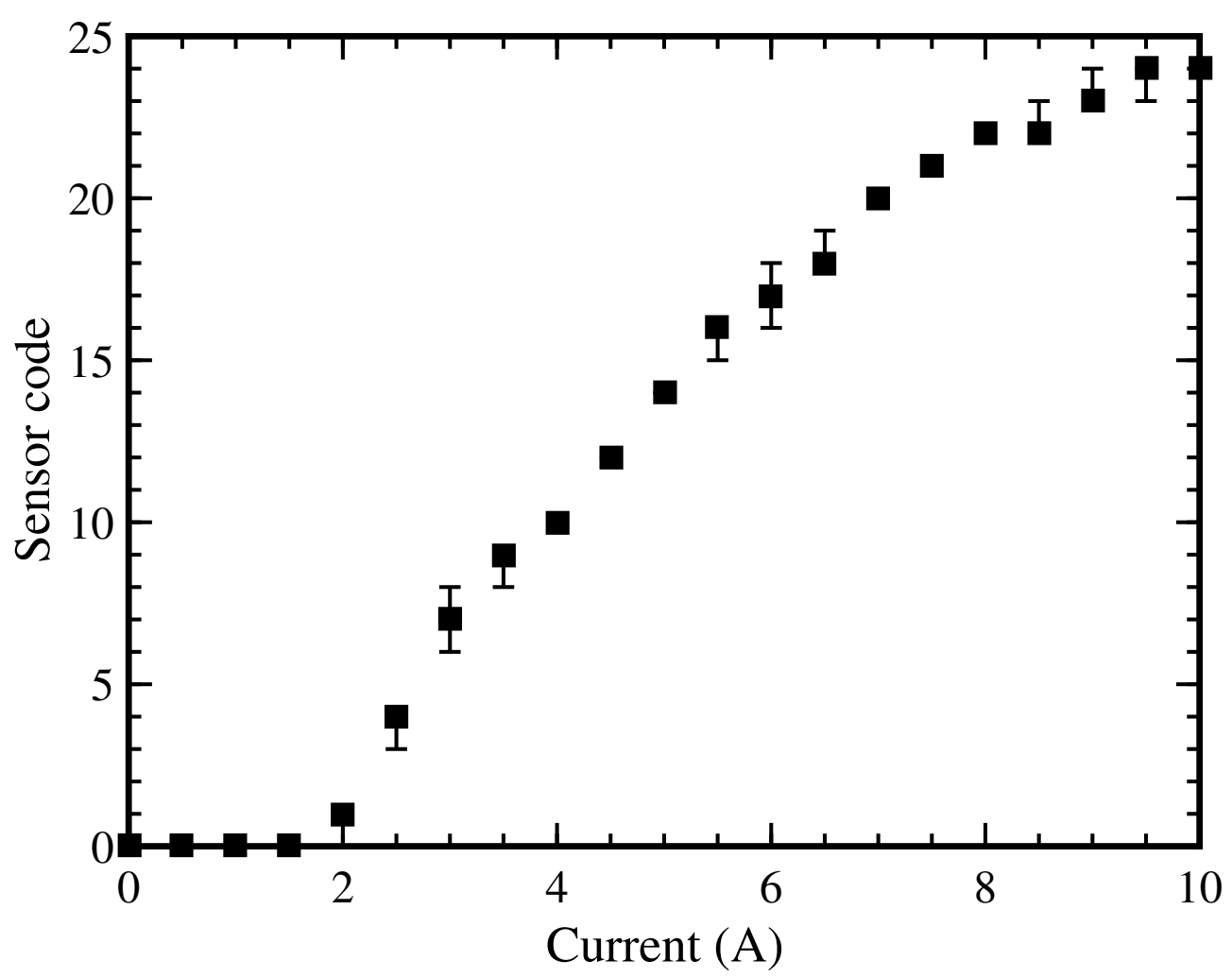

Fig. 11. Measured sensor code of the proposed RFID ac current sensor at $868 \mathrm{MHz}$.

of distance on the tag response, the tag system was placed at different distance intervals from the reader antenna. At $2 \mathrm{~cm}$ the tag response changed by approximately 3 sensor values as compared to the tag response at $30 \mathrm{~cm}$ distance at $868 \mathrm{MHz}$. It was observed that at $8 \mathrm{~cm}$ the tag response became stable i.e. same as the tag response at $30 \mathrm{~cm}$ distance. Additionally, as the tag returns RSSI data, the reader could be programmed to compensate for non-linearity at high signal strengths.

The input impedance of the chip remains stable between $-40^{\circ} \mathrm{C}$ to $85^{\circ} \mathrm{C}$. The average winding temperature of the $\mathrm{CT}$ does not exceed $85^{\circ} \mathrm{C}$ [24] which falls in the range of the chip operating temperature. Additionally, there is a large physical separation between the well insulated CT coil and the chip. Therefore, the minor heat that is generated in the coil will not conduct to the chip and the tag sensing performance will not be affected. Fig. 12 shows the maximum read range of the sensor to be 3.5 to $4 \mathrm{~m}$ across all readings. The lowest read range was recorded at 0 current because the tag is out of tuning range and the antenna power transfer coefficient $\tau$ 
decreases. The read range can be calculated by using the well-known Friis free-space formula.

$$
r_{\text {max }}=\frac{c}{4 \pi f} \cdot \sqrt{\frac{E I R P \cdot G_{r} \cdot \tau}{P_{I C}}}
$$

where $c$ is the speed of light and $f$ is operating frequency. EIRP is the equivalent isotropic radiated power, depending on the local regulation (3.28 W in Europe and $4 \mathrm{~W}$ in the US), $G_{r}$ is the tag antenna gain and $\tau$ is $1-|S|^{2}$, where $S$ is referring to the reflection coefficient. $P_{I C}$ represents the sensitivity of the tag chip. Simulation of the tag antenna mounted directly on the current detector unit showed the tag realized gain (including input reflection loss) to be -3.95 $\mathrm{dBi}$, and the $S_{11}$ value at $868 \mathrm{MHz}$ was $-1.6 \mathrm{~dB}$ before the tag autotune to improve the match. For the specified chip turn on power of $-16 \mathrm{dBm}$, the calculated read range was $4.6 \mathrm{~m}$ which agrees well with measurement. Purposely, the tag resonance frequency was slightly shifted from $868 \mathrm{MHz}$ to effectuate maximum possible sensing range (0-31) of the tag at $868 \mathrm{MHz}$. The tag resonance frequency was compensated by changing the inductors value to $47 \mathrm{nH}$ of the tuning circuit as inductance influences the tag resonance frequency.

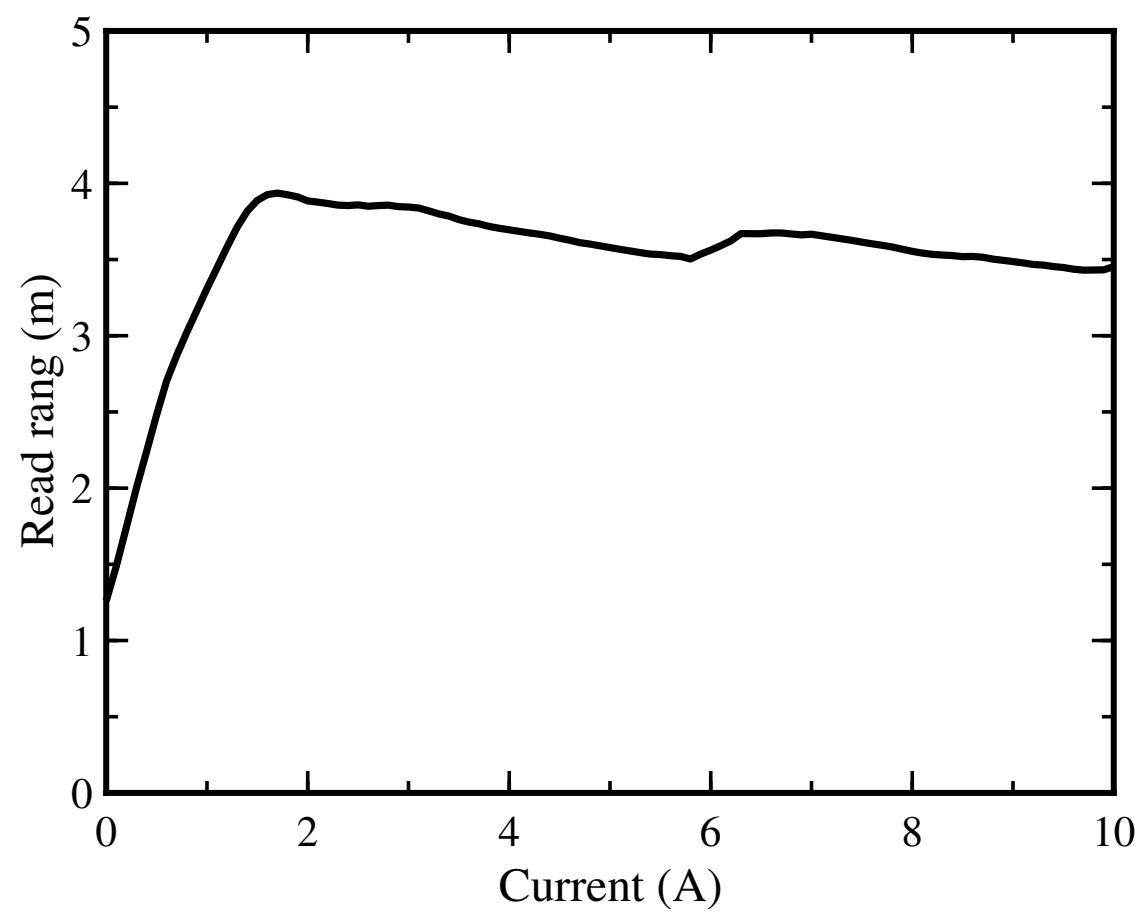

Fig. 12. Read range of the proposed RFID ac current sensor at $868 \mathrm{MHz}$. 


\section{Rise and Fall Time}

To assess if the tag sensor sample rate was sufficient to track a switching response in the test load, the electrical load was switched at $0.5 \mathrm{~Hz}$ by a Solid State Relay (SSR) [25] controlled by a microcontroller and linked to the ThingMagic M6e RFID reader [26] as shown in Fig. 13. A custom software application on the ThingMagic API synchronised the Tag Memory read function with the SSR toggle status obtained from the microcontroller serial output. This enabled the logging of the rise and fall time of the load current. The time taken by the waveform to reach $90 \%$ of the peak was defined as the rise time under loaded (ON) condition, and the fall time for the unloaded (OFF) condition was the period taken to fall $90 \%$ from the maximum. Fig. 14 shows the switched waveform obtained from the tag with rise and fall times of $368 \mathrm{~ms}$ and $276 \mathrm{~ms}$ respectively. Occasionally, the reader will drop an initial read cycle and if this occurs, reading the subsequent data packet takes longer according to the RFID EPC GEN2 protocol. As a consequence, the sample period of the sensor varied between 30 and $61 \mathrm{~ms}$, though this was adequate to capture the switching waveform. There is also an inherent delay between requesting and receiving samples which varies from a few to a dozen milliseconds. This is caused by factors including the SSR response time of $10 \mathrm{~ms}$, the time taken by the RFID reader to detect, read and request the sensor data and also the time taken between sending and receiving commands from computer to microcontroller and vice versa via a USB serial port.

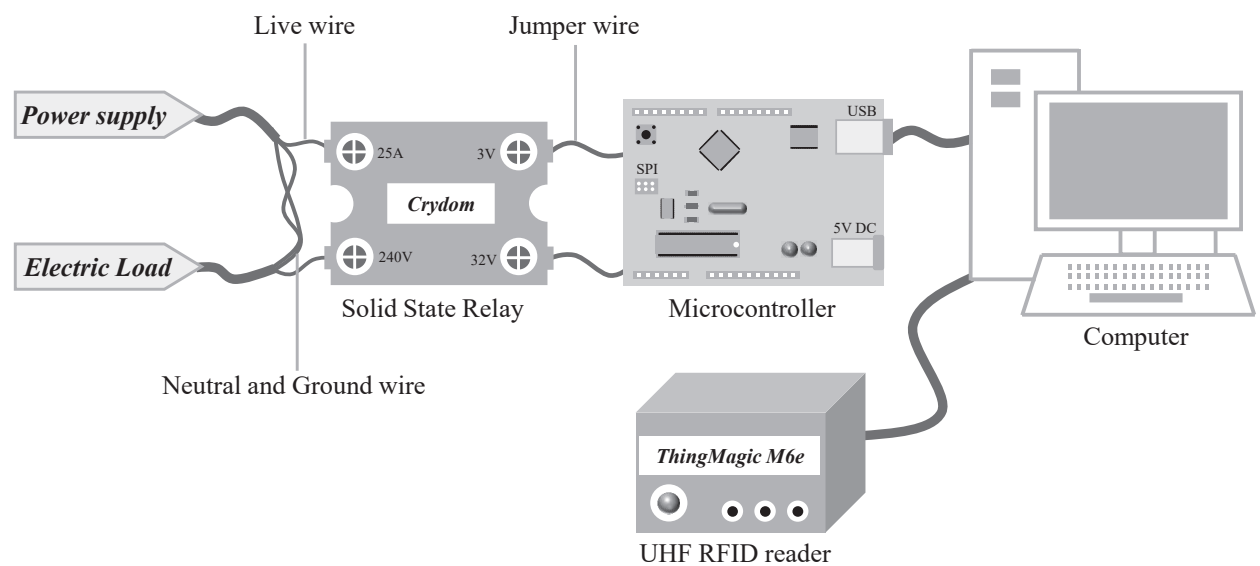

Fig. 13. Sensing set up for measuring the rise and fall time of a switching load current. 


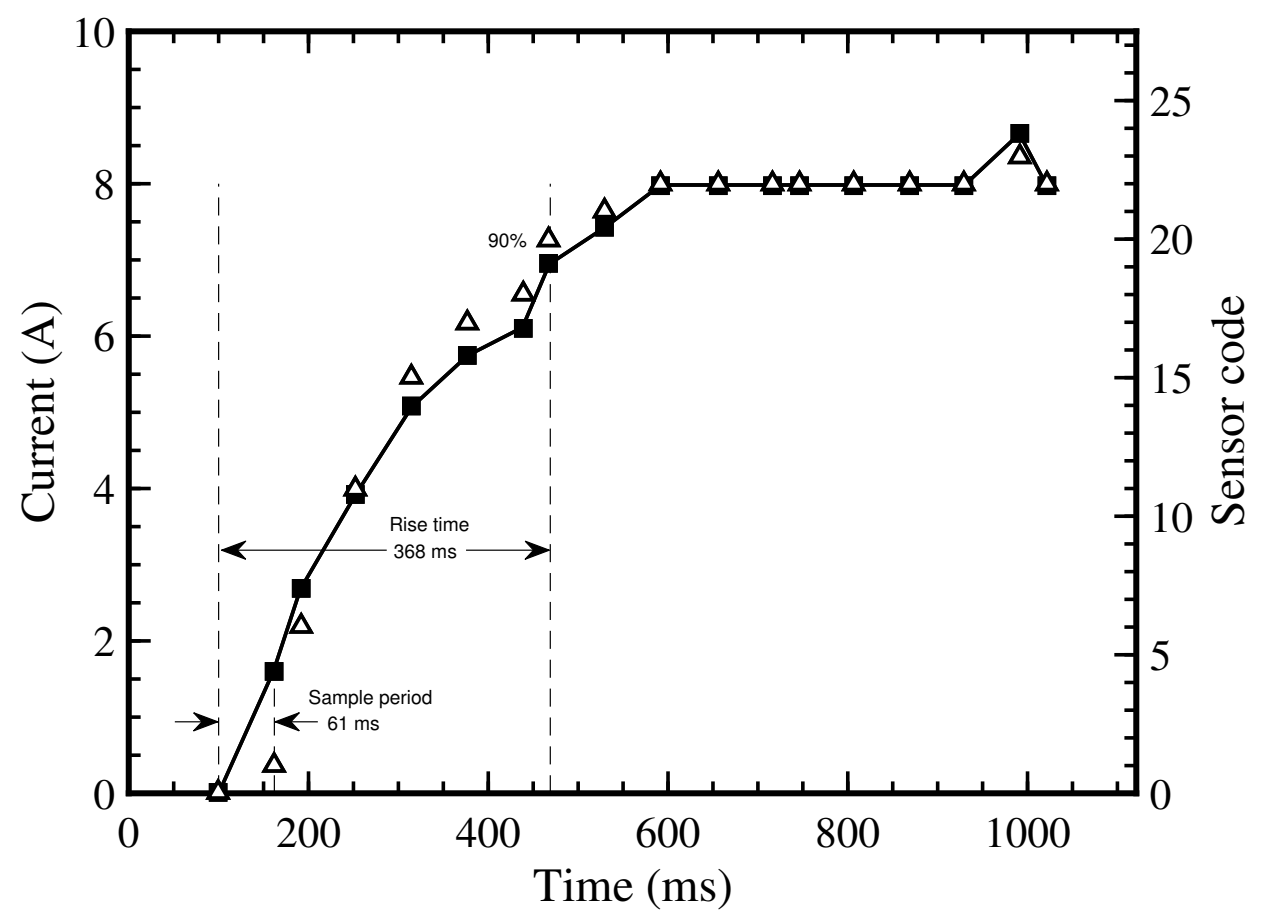

(a)

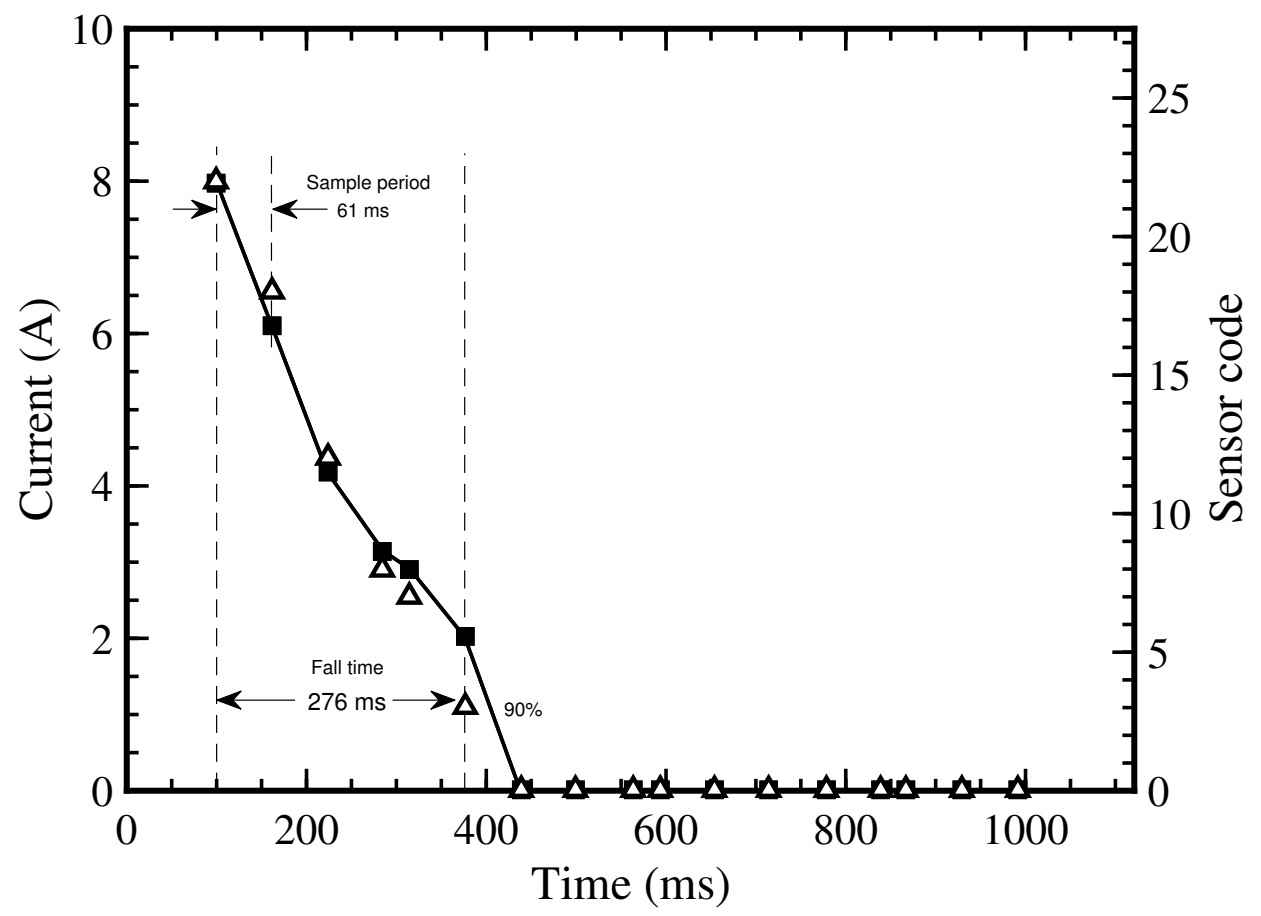

(b)

Fig. 14. Rise time and fall time of the RFID ac current sensor at $868 \mathrm{MHz}$. Triangle markers represent sensor code measured by the sensor and black solid line with square markers represent load current reconstructed from sensor code. (a) Rise time (368 ms); (b) Fall time (276 ms). 


\section{E. Sensor comparison}

Table 1 shows the comparison of the proposed tag system with the commercial alternative ac current meters. The total cost of the tag device is inclusive of the cost of CT (\$45.00) and the estimated cost of the tag (\$5.00). In order to be cost-effective for a domestic setting the RFID reader should be redeveloped for domestic use and price range.

TABLE I

COMPARISON BETWEEN THE PROPOSED TAG SYSTEM AND COMMERCIAL ALTERNATIVE AC CURRENT METERS IN TERMS OF COMMUNICATION, COST AND POWER TYPE.

\begin{tabular}{lllll}
\hline \hline Ref. & Sensors & Communication & Cost $(\$)$ & Power type \\
\hline$[27]$ & SCT-125W-100 by Magnelab & IEEE 802.15.4, 2.4 GHz technology & 245.00 & Passive \\
{$[28]$} & IoT long range wireless ac current monitor & DigiMesh protocol & 179.95 & Active \\
{$[29]$} & Alta wireless ac current meter - 20 A - AA & Monnit proprietary sensor protocol & 150.00 & Active \\
& battery powered & & 98.00 & Passive \\
{$[30]$} & Pressac current sensor: one channel & Enocean wireless radio protocol & 50.00 & Passive \\
\hline & Proposed tag system & Radio frequency Identification & & \\
\hline \hline
\end{tabular}

\section{CONCLUSION}

A new RFID current sensor using an electronic tuning technique has been developed and experimentally tested with an electrical load. The device can detect ac current in the range of 1.5-10 A from an ac electrical cable in smart power monitoring systems. The rise and fall times of the sensing tag is $368 \mathrm{~ms}$ and $276 \mathrm{~ms}$ respectively. The suggested mechanism could be modified for detecting currents lower than 1.5 A or higher than $10 \mathrm{~A}$ depending on the requirements of an application by altering the transformer turns ratio and tuning circuit. The proposed device is passive with a read range of about $4 \mathrm{~m}$ and is cost effective for multiple appliance monitoring through a single reader as opposed to more accurate, but relatively expensive single point smart meters.

In this work, we have not considered the wider metering system that the tag sensor would be connected to. It may be necessary to develop new metering methods in order to log and display the simultaneous consumption of multiple appliances. The tags could be deployed in modified plugs or as inline cable devices. The reader would be centrally mounted with a view of the tags to be read. 
Future work includes creating a new tag antenna design to be integrated with the CT housing in a compact form.

\section{ACKNOWLEDGMENT}

The INCASE project is part-funded by the European Commission as part of the 2Seas Intereg V Programme. We would like to thank Simon Jakes for fabricating the tags.

\section{REFERENCES}

[1] International Energy Agency (2019, Feb. 13). World energy outlook 2018: The gold standard of energy analysis [Online]. Available: https://www.iea.org/weo2018/

[2] United States Environmental Protection Agency (2019, Feb. 13). Greenhouse gas emissions [Online]. Available: https://www.epa.gov/ghgemissions/sources-greenhouse-gas-emissions

[3] Government UK, "Smart meters: unlocking the future," [Online], Dec. 31, 2018. Available: https://www.gov.uk/government/publications/smart-meters-unlocking-the-future/smart-meters-unlocking-the-future

[4] M. A. Ziai and J. C. Batchelor, "Smart radio-frequency identification tag for diaper moisture detection," IET Healthc. Technol. Lett., vol. 2, no. 1, pp. 18-21, 2015.

[5] A. A. Babar, S. Manzari, L. Sydanheimo, A. Z. Elsherbeni and L. Ukkonen, "Passive UHF RFID tag for heat sensing applications," IEEE Trans. Antennas Propag., vol. 60, no. 9, pp. 4056-4064, Sept. 2012.

[6] M. A. Ziai and J. C. Batchelor, "Multi-state logging freeze detection passive RFID tags," IEEE Trans. Antennas Propag., vol. 62, no. 12, pp. 6406-6411, Sep. 2014.

[7] A. Louzir, R. Kumar and J. -Y. L. Naour, "RFID based solution for the sensing of home electrical devices activity," in Proc. ISAP, Okinawa, Japan, 2016, pp. 1002-1003.

[8] R. Kumar, A. Louzir and J. -Y. L. Naour, "RFID helix antenna on power cord for the sensing of home electrical devices activity," in Proc. EUCAP, Paris, France, 2017, pp. 2554-2558.

[9] R. Kumar, A. Louzir and J. -Y. L. Naour, "RFID tag coupled with a magnetic sensor for wireless sensing of home electrical devices," in Proc. EuMC, Madrid, Spain, 2018, pp. 981-984.

[10] K. -L. Chen, Y. -R. Chen, Y. -P. Tsai, N. Chen "A novel wireless multifunctional electronic current transformer based on ZigBee-based communication,” IEEE Trans. Smart Grid., vol. 8, no. 4, pp. 1888-1897, Mar. 2016.

[11] CLEODE (2019, Feb. 10). ZPlug Boost [Online]. Available: http://www.cleode.fr/en/produits.php?page=zplug

[12] S. DeBruin, B. Campbell, and P. Dutta, "Monjolo: an energy-harvesting energy meter architecture," in SenSys'13, Roma, Italy, Nov. 2013, pp. 18:1-18:14.

[13] T. Hosseinimehr, and A. Tabesh, "Magnetic field energy harvesting from AC lines for powering wireless sensor nodes in smart grids," Opt. Lett., vol. 63, no. 8, pp. 4947-4954, Feb. 1986.

[14] M. C. Caccami and G. Marrocco, "Electromagnetic modeling of self-tuning RFID sensor antennas in linear and nonlinear regimes," IEEE Trans. Antennas Propag., vol. 66, no. 6, pp. 2779-2787, Jun. 2018.

[15] K. Zannas, H. El Matbouly, Y. Duroc and S. Tedjini, "Self-tuning RFID tag: a new approach for temperature sensing," IEEE Trans. Microw. Theory Techn., vol. 66, no. 12, pp. 5885-5893, Dec. 2018

[16] Smartrac-group (2019, Feb. 10). AN-FAM1601: passive sensors technical guide [Online]. Available: https://www.smartracgroup.com 
[17] Magnelab (2019, Feb. 10). DCT-0016-10/10Vdc split-core current sensor [Online]. Available: http://www.magnelab.com/products/ac-splitcore-current-sensor-dct-0016-100/

[18] Skyworks (2019, Feb. 5). SMV2019 series hyperabrupt junction tuning varactors [Online]. Available: https://www.skyworksinc.com/Product/668/SMV2019_Series

[19] Skyworks, "SMV2019 to SMV2023 series: hyperabrupt junction tuning varactors," SMV2019 to SMV2023 varactors datasheet, Aug. 2015.

[20] Axzon (2019, Feb. 10). RFM2100 wireless flexible moisture sensor [Online]. Available: https://axzon.com/rfm2100wireless-flexible-moisture-sensor/

[21] CST Microwave Suite 2017. [Online]. Available: https://www.cst.com

[22] Voyantic (2019, Feb. 10). Tagformance Pro [Online]. Available: http://voyantic.com/products/tagformance-pro

[23] Brennenstuhl smart technology (2018, Feb. 9). Primera-line wattage and current meter PM 231 E [Online]. Available: https://www.brennenstuhl.co.uk/en-GB/products/adapter-plugs/primera-line-wattage-and-current-meter-pm-231-e-gb

[24] IEEE standards association (2019, Aug. 25). IEEE C57.13-2016 - IEEE Standard Requirements for Instrument Transformers [Online]. Available: https://standards.ieee.org/standard/C57_13-2016.html

[25] Crydom GN Series (2019, Feb. 10). Panel mount easy pick: 84134210 [Online]. Available: http://www.crydom.com/en/products/panel-mount/easy-pick/84134210/

[26] Jadak tech (2019, Feb. 10). ThingMagic M6e RFID [Online]. Available: https://www.jadaktech.com/products/rfid/embeddeduhf-rfid-readers/mercury6e-m6e/

[27] Magnelab (2019, Aug. 26). SCT-125w-100 by Magnelab [Online]. Available: https://www.magnelab.com/products/wirelessac-current-sensor-system-sct-125w/

[28] NCD (2019, Aug. 26). IoT long range wireless ac current monitor [Online]. Available: https://store.ncd.io/product/wirelessac-current-monitor/

[29] Monnit (2019, Aug. 26). Alta wireless ac current meter - 20 A - AA battery powered [Online]. Available: https://www.monnit.com/Product/MNS2-9-W2-CM-020/

[30] Pressac (2019, Aug. 26). Current sensor: one channel [Online]. Available: https://www.pressac.com/current-monitoringsensors/

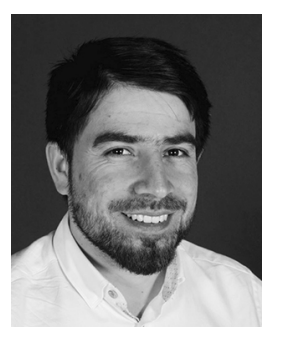

Irfan Ullah received the BEng (Hons) degree in Electronic and Communications Engineering in 2016 at University of Kent, Canterbury, U.K., where he is currently working toward his Ph.D. degree in Electronic Engineering.

His current research interests include small antennas, passive UHF RFID tag and passive sensing devices. 


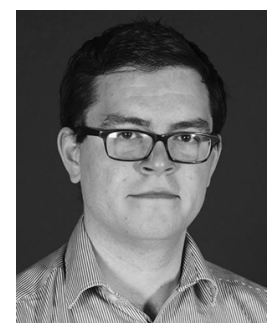

Robert Horne received the MEng (Hons) and Ph.D. degrees from the University of Kent, Canterbury, U.K., in 2012 and 2017 respectively.

From 2016, he joined Evidentia limited as a research director where he worked on embedded skin electronics. From 2017, he became a Research Associate with the School of Engineering and Digital Arts, University of Kent where he currently works on embedded solutions for epidermal ultra-high frequency radio frequency identification sensor platforms.

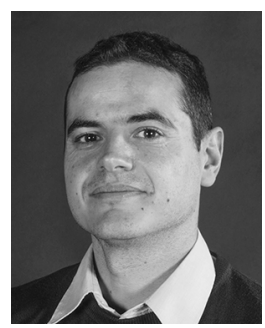

Benito Sanz-Izquierdo received the B.Sc. degree from the University of Las Palmas de Gran Canaria, Las Palmas, Spain, and the M.Sc. and Ph.D. degrees from the University of Kent, Canterbury, U.K., in 2002 and 2007, respectively.

From 2003 to 2012, he was a Research Associate with the School of Engineering and Digital Arts, University of Kent, and became a Lecturer of electronic systems in 2013. In 2012, he joined Harada Industries Ltd., U.K., where he developed novel antennas for the automotive industry. His current research interests include multiband antennas, wearable microwave devices, substrate-integrated waveguides components, electromagnetic bandgap structures, and frequency selective surfaces.

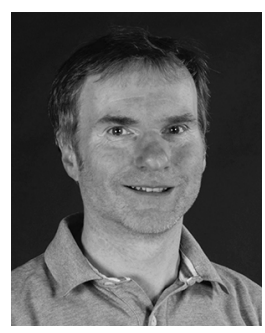

John C. Batchelor (S'93-M'95- SM'07) received the B.Sc. and Ph.D. degrees from the University of Kent, Canterbury, U.K., in 1991 and 1995, respectively.

He was a Research Assistant with the Electronics Department, University of Kent, from 1994 to 1996, where he became a Lecturer of electronic engineering in 1997. He currently leads the Antennas Group, University of Kent, where he is also a Professor of antenna technology. His current research interests include ultra-high frequency radio frequency identification tag design, passive sensing, bodycentric antennas, printed antennas, compact multiband antennas, electromagnetic bandgap structures, and long wavelength frequency-selective surfaces. 